\title{
Screening and biological activities of pedopeptins, novel inhibitors of LPS produced by soil bacteria
}

\author{
Shiho Kozuma ${ }^{1}$, Yuki Hirota-Takahata ${ }^{2}$, Daisuke Fukuda ${ }^{2}$, Nahoki Kuraya ${ }^{3}$, Mutsuo Nakajima ${ }^{3}$ \\ and Osamu Ando ${ }^{4}$
}

Lipopolysaccharide (LPS) is a strong endotoxin and is delivered to the cell surface signaling receptor, Toll-like receptor 4 and MD-2 complex, via soluble cluster of differentiation (CD) 14 or membranous CD14, resulting in the induction of the inflammatory response. To obtain new compounds that block LPS binding to CD14, we designed a high-throughput screening based on time-resolved intermolecular fluorescence resonance energy transfer. This cell-free screening system successfully led to the discovery of novel inhibitors of LPS-CD14 interaction from the library of the secondary metabolites of microorganisms. We identified the novel compounds pedopeptin A, B and C from a culture broth of Pedobacter sp. SANK 72003. Pedopeptins blocked LPS binding to CD14 in vitro with $\mathrm{IC}_{50}$ values of 20,11 and $47 \mathrm{~nm}$, respectively, and also inhibited LPS binding to the cells expressing CD14, leading to the suppression of cytokine production. Moreover, they showed antimicrobial activities against Escherichia coli with minimum inhibitory concentration ranging from 2 to $4 \mu \mathrm{g} \mathrm{ml}-1$.

The Journal of Antibiotics (2014) 67, 237-242; doi:10.1038/ja.2013.121; published online 27 November 2013

Keywords: CD14; FRET; lipopolysaccharide; pedopeptin

\section{INTRODUCTION}

Lipopolysaccharide (LPS) is one of the potent pathogen-associated molecular patterns and is a component of the gram-negative bacterial outer membrane. The mechanism by which LPS acts on the cells has been well elucidated. First, LPS forms a complex with LPS-binding protein (LBP), a plasma protein that helps LPS bind to its receptor cluster of differentiation (CD) 14 with high affinity. ${ }^{1}$ CD14 has two forms, soluble CD14 (sCD14) and membrane-anchored CD14 (mCD14) by glycosylphosphatidylinositol. ${ }^{2,3}$ The signaling receptor for LPS is Toll-like receptor (TLR) $4,{ }^{4}$ a type 1 transmembrane molecule with an adaptor molecule MD-2 at the extracellular domain. ${ }^{5}$ Both forms of CD14 transfer LPS-LBP to TLR4-MD-2, ${ }^{6}$ and cells are activated to produce various inflammatory mediators.

In the human immune system, LPS activates an innate immune system to fight against bacteria; however, surplus exposure to LPS can cause severe inflammation. For example, sepsis is a systemic inflammatory response syndrome with high mortality triggered by bacterial infection and consequent LPS release. ${ }^{7}$ Therefore, inhibition of LPS stimuli is a promising strategy for anti-sepsis therapy.

To inhibit LPS-mediated activation, a variety of efforts have been made. Eritoran, an analog of the lipid A portion, which is one of the components of LPS and has the endotoxic nature of LPS, potently antagonizes LPS, although this did not meet the primary endpoint in the phase 3 clinical trial. ${ }^{8}$ Polymyxin B (PMB), a cyclic lipopeptide antibiotic (Figure 1) isolated from the culture broth of Bacillus polymyxa (currently known as Paenibacillus polymyxa) with antimicrobial activity in $1947^{9}$ directly binds to and neutralizes LPS. PMB potently inhibits the pathological activities of LPS in animal models, ${ }^{10-12}$ however, the use of PMB is limited to topical administration in humans due to its neural and renal toxicity. ${ }^{13}$ These results prompted us to conduct a screening to identify novel LPS inhibitors. Here, we describe the construction of a screening method of LPS inhibitors and the discovery of the novel compounds. We decided to screen the extract of natural product, especially the secondary metabolites of microorganisms, because some LPSbinding compounds were derived from them. The assay was based on intermolecular fluorescence resonance energy transfer (FRET) technology, ${ }^{14}$ which enables detection of the interaction between two molecules in a homogenous condition. We selected this method because the extracts of the natural products are miscellaneous and a cellular assay system is often needed to dilute the samples to a large extent without being affected by contaminant cytotoxic ingredients. We report in this paper the discovery of novel and potent LPS inhibitors named pedopeptin A, B and C from a culture broth of soil bacteria using a newly developed assay system, and that the disruption of LPS-CD14 interaction with pedopeptins leads to the inhibition of LPS-induced cellular response.

\section{RESULTS}

Binding of LPS to the CD14-expressing cells

It is reported that LPS-LBP is delivered or transferred to cell surface TLR4 by sCD14 or mCD14. To confirm the contribution of CD14, the

\footnotetext{
${ }^{1}$ Biologics Research Laboratories, Daiichi-Sankyo Co., Ltd., Tokyo, Japan; ${ }^{2}$ Drug Discovery and Biomedical Technology Unit, Daiichi-Sankyo RD Novare Co., Ltd., Tokyo, Japan; ${ }^{3}$ Exploratory Laboratories, Daiichi-Sankyo Co., Ltd., Tokyo, Japan and ${ }^{4}$ Oncology Research Laboratories, Daiichi-Sankyo Co., Ltd., Tokyo, Japan Correspondence: Dr O Ando, Oncology Research Laboratories, Daiichi-Sankyo Co., Ltd., 1-2-58 Hiromachi, Shinagawa-ku, Tokyo 140-8710, Japan. E-mail: ando.osamu.jy@daiichisankyo.co.jp
}

Received 1 April 2013; revised 19 September 2013; accepted 25 October 2013; published online 27 November 2013 


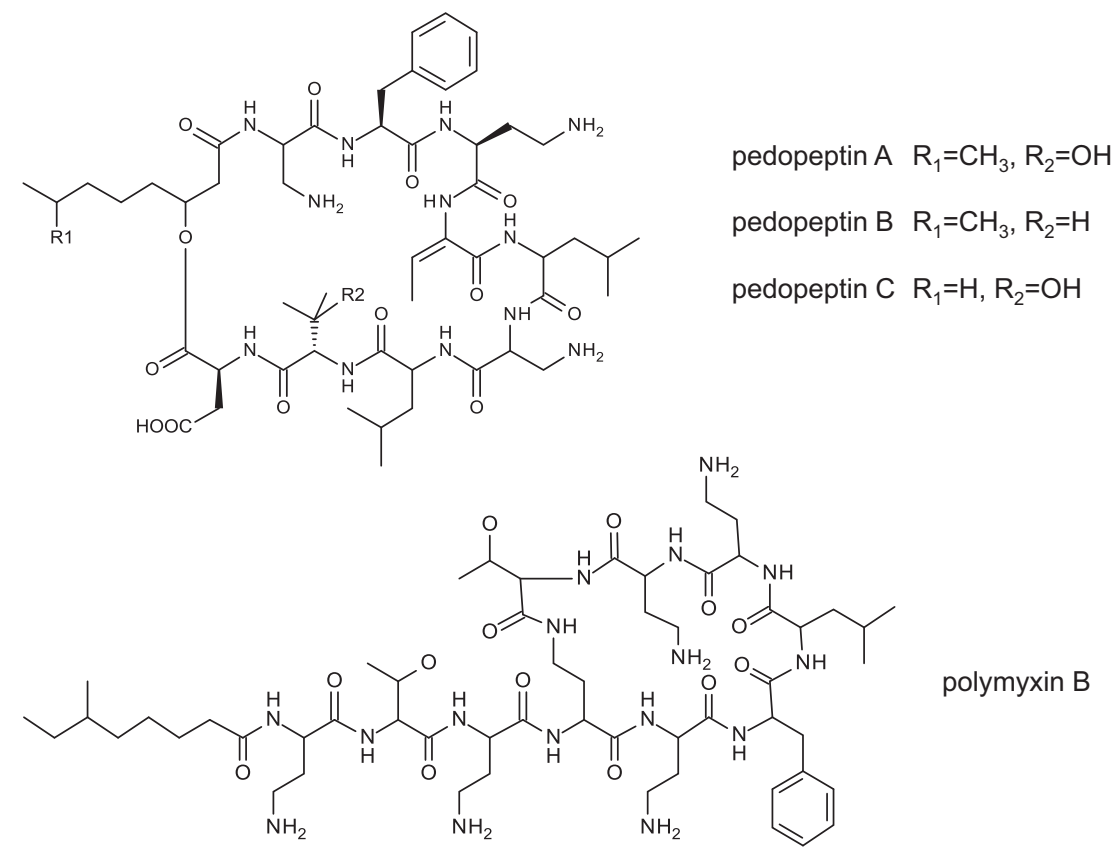

Figure 1 Chemical structures of pedopeptin $\mathrm{A}-\mathrm{C}$ and $\mathrm{PMB}$.
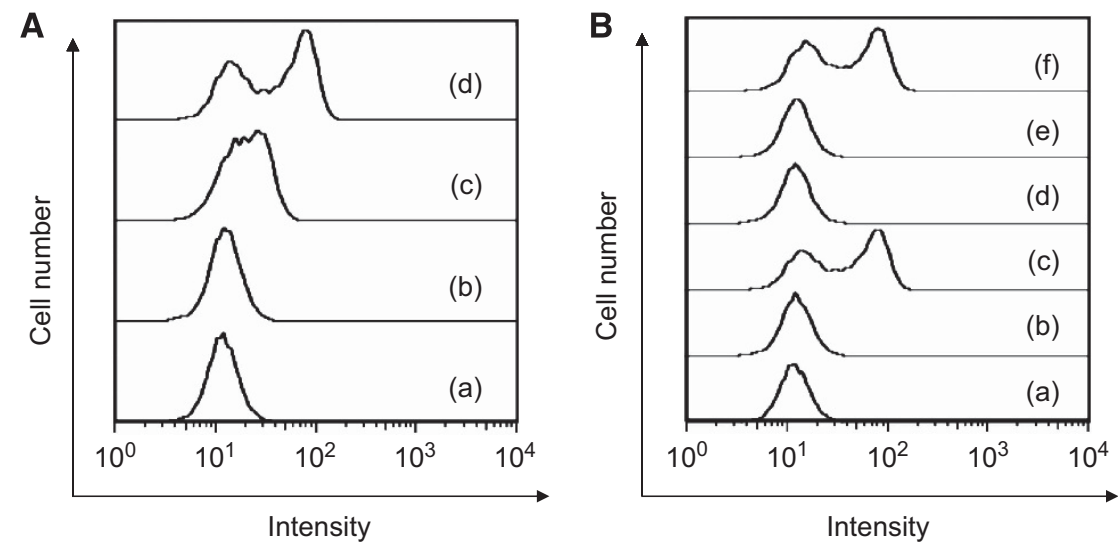

Figure 2 CD14 expression contributes LPS binding. (A) HEK293T cells were transiently transfected with the control vector (a) or mCD14 plasmid (b-d). The cells were incubated with $0 \mathrm{ng} \mathrm{ml}^{-1}$ (b), $200 \mathrm{ng} \mathrm{ml}^{-1}$ (c) and $1000 \mathrm{ng} \mathrm{ml}^{-1}$ (d) of FITC-LPS, and binding was detected using flow cytometry. (B) The effect of additional expression of TLR4 and MD-2 to CD14 on LPS binding was determined. Mock cells were not treated (a). Mock (b), CD14 (c), TLR4 (d),

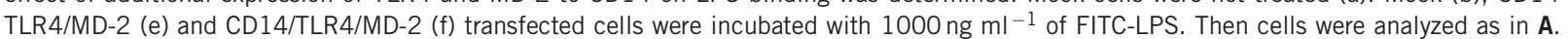

binding of fluorescein isothiocyanate-labeled LPS (FITC-LPS) to CD14-expressing cells was tested using flow cytometry. We compared FITC-LPS binding with the cell surface between mCD14-transfected and untransfected HEK293T cells. The FITC intensity was increased in mCD14-expressing cells in LPS dose-dependent manner, indicating that the expression of $\mathrm{mCD} 14$ enables LPS binding to the cell surface of HEK293T (Figure 2A).

As the LPS-LBP-CD14 complex requires TLR4 and MD-2 to transmit LPS signaling, ${ }^{6}$ we next examined whether the expression of TLR4 and MD-2 enhanced LPS binding to the cells. Additional expression of TLR4 and MD-2 to CD14 did not change FITC-LPS binding (Figure $2 \mathrm{~B}$ ). Figure $2 \mathrm{~B}$ also showed that the cells expressing TLR4 or TLR4/MD-2 did not bind to FITC-LPS. These results support the previous reports that CD14 participates in LPS binding to the cell surface; thus, we selected LPS-CD14 interaction as the readout for LPS inhibitor screenings.

\section{Construction of LPS-CD14 FRET assay}

Although the flow cytometric analysis of LPS-CD14 interaction gave rise to a clear signal, it did not allow us to reduce the assay process and scale, and was not suitable for random screening of natural product samples. Therefore, we developed a cell-free assay method applicable to the screening of the inhibitors of LPS binding to CD14.

FRET assay was employed, and LANCE Eu and XL665 were used as fluorophores of a donor and an acceptor, respectively. sCD14 instead of mCD14 was tagged with myc-epitope, and myc-tagged CD14 was detected with anti-myc antibody labeled with LANCE Eu. Biotin-LPS was detected with SA-XL665. When mixed in a buffer containing serum that supplied LBP, LPS and CD14 in proximity bore an energy transfer, and a fluorescent signal was detected (Figure 3a). LPS- and sCD14-dose-dependent signals were observed (data not shown), and optimal concentrations and conditions were determined as described in MATERIALS AND METHODS. To confirm that our TR-FRET 

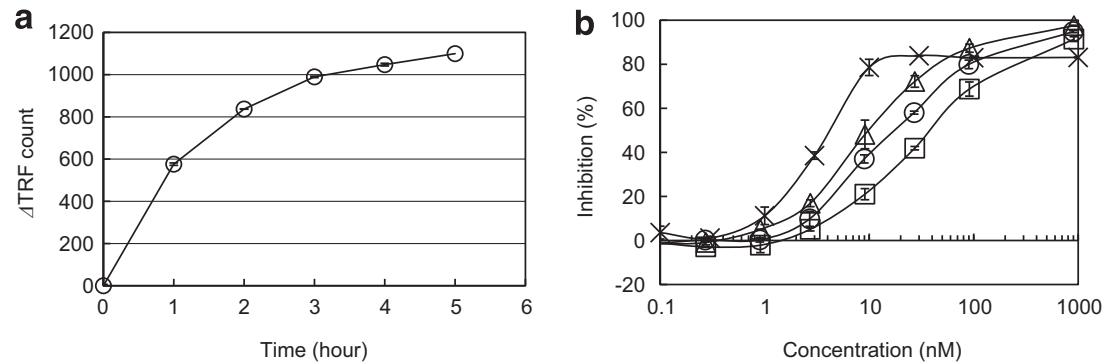

Figure 3 LPS-CD14 interaction monitored using TR-FRET assay. (a) Biotin-LPS and myc-CD14 were incubated with SA-XL665 and Europium Cryptatelabeled anti-myc antibody for the indicated hours at room temperature and TR-FRET was measured. (b) TR-FRET assay was performed with various concentrations of pedopeptin A (circles), pedopeptin B (triangles), pedopeptin C (squares) and PMB (crosses).

assay system was appropriate for the screening of LPS-binding inhibitors, the effect of PMB was examined. As shown in Figure 3b, $\mathrm{PMB}$ inhibited TR-FRET in a dose-dependent manner with an $\mathrm{IC}_{50}$ value of $3.6 \mathrm{~nm}$, ensuring relevancy of our TR-FRET assay system.

\section{Screening for inhibitors of LPS binding to CD14}

We conducted high-throughput screening with the TR-FRET assay system mentioned above and screened 61500 natural product samples using 384-well plates. Then inhibitory samples were subjected to subsequent assay to eliminate Limulus-positive samples. As a result, potent inhibitory activity was detected in a fermentation broth of Pedobacter sp. SANK 72003. The activity was divided into three fractions, and three novel compounds were isolated and designated as pedopeptins. The structures are shown in Figure 1. Details of the taxonomy of the producing organism, isolation and the structure elucidation of the active components will be described in HirotaTakahata's manuscript. ${ }^{15}$

\section{Inhibition of LPS binding to CD14 by pedopeptins}

To characterize pedopeptins, we tested their inhibitory activities on LPS-CD14 binding in different concentrations. Pedopeptins showed potent inhibitory activities in a concentration-dependent manner (Figure $3 \mathrm{~b}$ ), and their $\mathrm{IC}_{50}$ values were 20,11 and $47 \mathrm{~nm}$ for pedopeptin A, B and C, respectively. Their potencies were similar and pedopeptin $\mathrm{B}$ was the strongest. In the same assay system, PMB showed the $\mathrm{IC}_{50}$ value $3.6 \mathrm{~nm}$.

Inhibition of LPS binding to the cell surface by pedopeptins We examined whether pedopeptins inhibited LPS binding to the cells expressing CD14. As shown in Figure 4, pedopeptins blocked the binding of FITC-LPS to the cell surface. At indicated concentrations, they did not show any cytotoxicity. These results show that pedopeptins inhibit LPS binding not only to sCD14 but also membranous CD14 on the cell surface, expecting the potential to block LPS-triggered cellular responses.

Inhibition of LPS-induced TNF- $\alpha$ production in PMA-treated U937 cells by pedopeptins

Next, we tested whether the blockade of LPS binding was enough to inhibit cellular response against LPS stimuli. Cells produce various cytokines in response to LPS recognition and U937, a human monocyte cell line, is known to differentiate into macrophage-like cells by treatment with PMA and to produce TNF- $\alpha$ upon LPS stimulation. We employed this system and demonstrated that pedopeptins inhibited TNF- $\alpha$ production in dose-dependent manners and $\mathrm{IC}_{50}$ values were ranged from 0.08 to $0.33 \mu \mathrm{M}$ (Figure 5). Their inhibitory effects were not accompanied with cytotoxicity. As in the case of LPS-CD14 interaction, pedopeptin B was the most potent and PMB showed stronger inhibition than pedopeptins.

\section{Effects on cell viability by pedopeptins}

Pedopeptins did not show any cytotoxicites at the concentrations of LPS inhibition in binding and cytokine assay as described above. Then we tested their effects on cell viability at higher concentrations than the effective dose. As a result, pedopeptin A and pedopeptin B showed cytotoxicity at 100 and $30 \mu \mathrm{g} \mathrm{ml}^{-1}$, respectively (Figure 6). $\mathrm{PMB}$ did not show apparent cytotoxicity at $100 \mu \mathrm{g} \mathrm{ml}^{-1}$.

\section{Antimicrobial activity of pedopeptins}

PMB has been known to have bactericidal activities especially against Gram-negative bacteria. To examine the antimicrobial potencies of pedopeptins, MICs of pedopeptins were determined using E. coli and Staphylococcus aureus strains as antibacterial indicators. Pedopeptins showed good antimicrobial activities against two E. coli strains with MIC ranging from 2 to $4 \mu \mathrm{g} \mathrm{ml}^{-1}$, and pedopeptin B also showed strong antibacterial activity against Gram-positive bacteria $S$. aureus at lower concentration than pedopeptin A, C and PMB (Table 1).

\section{DISCUSSION}

LPS exerts its pathological activities by binding to the cell surface and the following signal activation. There are many ways to prevent LPSinduced cellular activation, for example, LPS neutralization, inhibition of signaling cascades, trapping released cytokine and chemokine, blocking their receptors, and so on. Thus far, various efforts have been made to treat LPS-induced systemic inflammatory response, but it could not easily be controlled by modifying some outputs after LPS triggering, because they are synergistically augmented. From this point of view, pharmacological inhibition of LPS binding to its target cells such as monocytes, macrophages and endothelial cells, is considered to be effective in blocking the immunostimulatory activities of LPS. This is because we focused on LPS binding and aimed at LPS-binding inhibitor screening. We showed that CD14 expression on the cell surface contributed LPS binding (Figure 2) and decided to screen inhibitors of LPS-CD14 interaction. It is known that some LPS, called R-LPS, does not always need CD14 for its signaling, ${ }^{16}$ but it is generally considered that CD14 is an important molecule to detect LPS because CD14-dependent responses are of high affinity compared to CD14-independent ones. ${ }^{17}$

To conduct high-throughput screening, a cell-free homogenous LPS-CD14-binding assay using TR-FRET technology was developed. Complex components were prepared in two solutions, one containing LPS and an anti-CD14 reagent, and the other containing CD14 and 
A

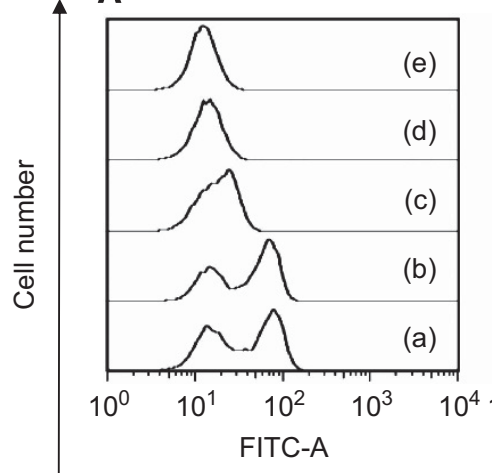

B

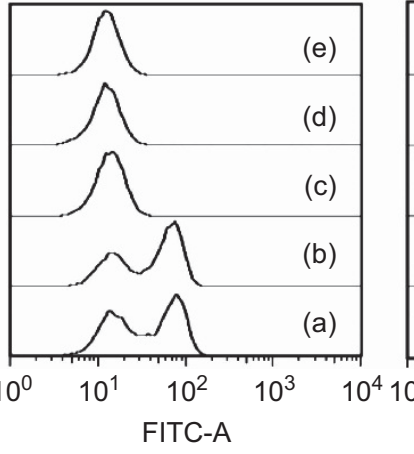

C

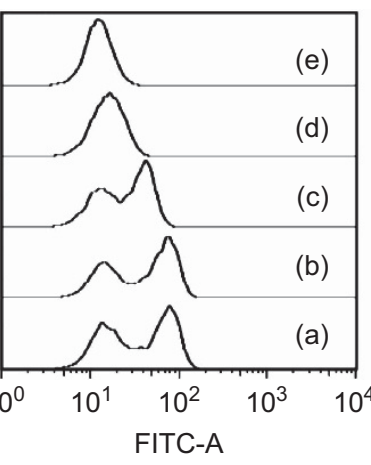

D

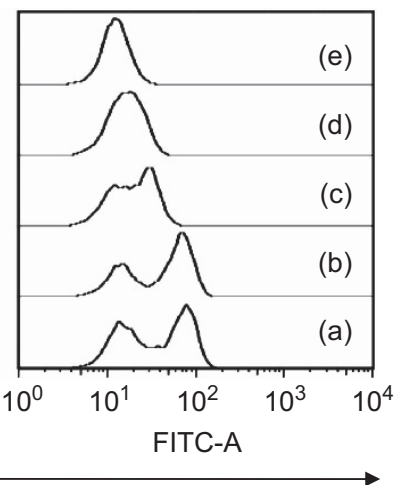

Intensity

Figure 4 Inhibition of LPS binding to the cell surface by pedopeptin A-C. HEK293T cells expressing CD14 were treated with various concentrations of pedopeptin A (A), pedopeptin B (B), pedopeptin C (C) and PMB (D). The cells were treated with $0 \mu \mathrm{g} \mathrm{ml}-1$ (a), $0.1 \mu \mathrm{g} \mathrm{ml}-1$ (b), $1 \mu \mathrm{g} \mathrm{ml} \mathrm{l}^{-1}$ (c) and $10 \mu \mathrm{g}$ $\mathrm{ml}^{-1}$ (d) of pedopeptin or PMB with $1000 \mathrm{ng} \mathrm{ml}^{-1}$ of FITC-LPS. The chart (e) shows without FITC-LPS. FITC intensity was detected as described in Figure 2.

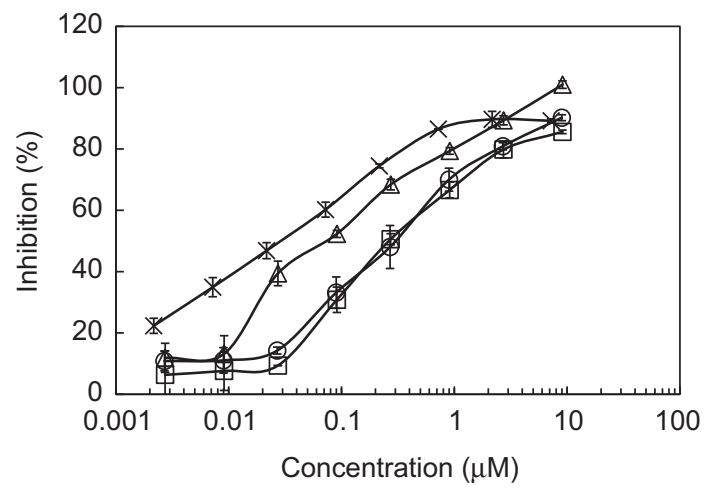

Figure 5 Inhibition of LPS-induced TNF- $\alpha$ production by pedopeptin A-C. U937 cells were differentiated into macrophage-like cells and treated with LPS as described in materials and methods. Pedopeptin A (circles), pedopeptin B (triangles), pedopeptin C (squares) and PMB (crosses) were treated with indicated concentrations for $4.5 \mathrm{~h}$ and the TNF- $\alpha$ production in the supernatant was measured.

an anti-LPS reagent. Therefore, the operation was easily performed in a 'mix and measure' manner. The remarkable advantage of this system was its simple handling, thus, allowing us to conduct high-throughput screening. As a screening source, we employed in-house natural product libraries, which we expect to contain physiologically active substances with varieties of structure, MW and character. During the analysis of active fractions, we eliminated lipid A analogs using the Limulus test, because bacterial extracts sometimes contain lipid A analogs and they might work as both antagonists and agonists in different assay conditions, ${ }^{18}$ which could lead to side effects. Finally, we succeeded in the discovery of novel LPS inhibitors from the secondary metabolites of soil bacteria and named them pedopeptins.

Structure analysis of pedopeptins revealed them to be cyclic depsipeptides. PMB is a cyclic peptide and directly binds to and neutralizes LPS. PMB has five cationic amino acids among a constituent of 10 amino acids, and the cationic character is important when interacting with anionic LPS. On the other hand, it is reported that cationic substances accumulate in proximal tubular epithelium and this may explain, at least partially, the development of nephrotoxicities. ${ }^{19} \mathrm{PMB}$ is potent in in vitro assays and in vivo animal models, but is not used systemically in humans because of its renal

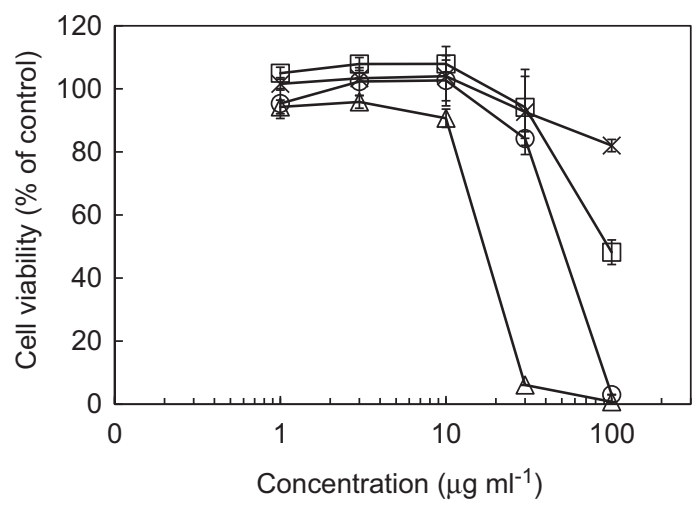

Figure 6 Effects on cell viability by pedopeptins. HEK293 cells were treated with indicated concentrations of pedopeptin A (circles), pedopeptin B (triangles), pedopeptin C (squares) and PMB (crosses) for $20 \mathrm{~h}$. Then cell viabilities were detected using Cell-Titer Glo according to the manufacturer's instructions.

Table 1 Antimicrobial activities of pedopeptin A, B, C and PMB

\begin{tabular}{lrrrc}
\hline & \multicolumn{4}{c}{$M I C\left(\mu g m l^{-1}\right)$} \\
\cline { 2 - 5 } Strain no. & $A$ & $B$ & $C$ & $P M B$ \\
\hline E. coli ATCC 47076 & 2 & 4 & 2 & 0.5 \\
E. coli NIHJ-JC2 & 2 & 4 & 2 & 0.25 \\
S. aureus ATCC 6538P & 32 & 4 & 64 & 64 \\
S. epidermis ATCC 14990 & 32 & 4 & 64 & 32
\end{tabular}

Abbreviations: MIC, minimum inhibitory concentration; PMB, Polymyxin B.

toxicity. Nevertheless, PMB is being considered as a resort against multi-resistant Gram-negative infections, which are serious problems in the clinics recently, and various novel derivatives of PMB are under study. For example, NAB739, which has a cyclic peptide portion identical to that of PMB but carries only three cationic amino acids, was reported to be eightfold less cytotoxic to renal cells and to have equal to eightfold higher $\mathrm{MIC}_{90}$ values. $^{20,21}$ We expect that pedopeptins, containing three cationic amino acids among a constituent of nine amino acids, have less renal toxicity than PMB 
as a result of their less cationic characters. Hence, we have significant interest in pedopeptins from the antibacterial aspect, as well as the potency of novel LPS inhibitor.

We showed pedopeptins inhibited LPS binding and LPS-induced cytokine release in in vtiro cell-based assays. They did not show cytotoxicity at effective concentrations $\sim 1-10 \mu \mathrm{g} \mathrm{ml} \mathrm{m}^{-1}$, but pedopeptin A and B showed cytotoxicity at 100 and $30 \mu \mathrm{g} \mathrm{ml} \mathrm{ml}^{-1}$, respectively. Gramicidin S, a cyclic peptide antibiotic with amphiphilic $\beta$-sheet conformation, is known to interact with the cell membrane and have high hemolytic activity. ${ }^{22}$ We noticed that cyclic amino-acid portion of pedopeptins seems to be amphiphilic because they have hydrophobic amino acids among basic ones in an almost alternating way as antimicrobial peptides have. ${ }^{23}$ This structural property of pedopeptins may lead to the capacity to interact with the lipid layer, which possibly results in the cytotoxicity at high concentration, and may also lead to the antimicrobial activities against Gram-positive bacteria, which are possibly governed by valine residue of pedopeptin B.

In conclusion, we discovered pedopeptins and showed their activities as LPS inhibitors, as well as antibiotics with broad spectrum. We will carry on in vivo studies of pedopeptins to evaluate their potentials as candidates of therapeutic agents and further analysis of the mechanism of action, especially whether they directly bind to LPS or not. In addition, we will continue further screening for a new series of inhibitors and these works will open up an opportunity for novel LPS inhibitors.

\section{MATERIALS AND METHODS}

\section{Materials}

LPS from E. coli J5 mutant lacking O-antigen was obtained from SigmaAldrich (St Louis, MO, USA) for biotinylation. Streptavidin-conjugated XL665 (SA-XL665) was obtained from Cisbio (Bedford, MA, USA). LANCE Europium (Eu)-labeled anti-c-myc antibody was obtained from PerkinElmer (Waltham, MA, USA). FITC-LPS (E. coli O111:B4) was obtained from Sigma-Aldrich (Tokyo, Japan). PMB sulfate was obtained from Sigma-Aldrich. HEK293 (CRL-1573) and U937 (CRL-1593.2) cells were obtained from American Type Culture Collection. Myc-tagged CD14 protein was prepared as a supernatant of COS-7 cells transiently transfected with sCD14-myc plasmid. Complete mini, protease inhibitor cocktail was obtained from Roche (Upper Bavaria, Germany). LPS from E. coli 055:B5 was obtained from SigmaAldrich for cell stimulation.

\section{Preparation of biotin-LPS}

LPS ( $1 \mathrm{mg}$ ) was dissolved in $0.1 \mathrm{~m}$ sodium acetate ( $\mathrm{pH} 5.0$ ) and oxidized in $1 \mathrm{ml}$ of $10 \mathrm{~mm} \mathrm{NaIO}_{4}$ solution on ice in the dark. After $30 \mathrm{~min}$, the reaction was terminated by the addition of $2 \mu \mathrm{l}$ of glycerol. Then, the reaction mixture was dialyzed with an $8000 \mathrm{MW}$ cutoff membrane in PBS at $4{ }^{\circ} \mathrm{C}$ overnight. Next, $0.1 \mathrm{ml}$ of $50 \mathrm{~mm}$ biotin-LC-hydrazide (Pierce, Rockford, IL, USA) was added into oxidized LPS and incubated for $2 \mathrm{~h}$ at room temperature with gentle mixing. The reaction mixture was dialyzed and stored at $4{ }^{\circ} \mathrm{C}$, and used as biotin-LPS.

\section{Flow cytometric analysis}

LPS binding to the cell surface was monitored by flow cytometry. Expression vectors for CD14, TLR4 and MD-2 were transfected into HEK293T cells using Lipofectamine 2000 (Invitrogen, Carlsbad, CA, USA). Two days after transfection, the cells were harvested and incubated with FITC-LPS for $1 \mathrm{~h}$ at $4{ }^{\circ} \mathrm{C}$ and washed with PBS. Then, FITC intensity was monitored with FACSCanto II (Becton Dickinson, Franklin Lakes, NJ, USA).

\section{LPS-CD14 binding assay}

LPS-CD14 binding was detected using time-resolved (TR) FRET. The buffer compositions were as follows: buffer A: $50 \mathrm{~mm}$ HEPES, pH 7.2, $100 \mathrm{ng} \mathrm{ml}^{-1}$
biotin-LPS, $120 \mathrm{ng} \mathrm{ml}^{-1}$ Eu-labeled anti-c-myc antibody, $0.2 \%$ fetal calf serum, $0.2 \%$ bovine serum albumin, one tablet of complete, mini. in $10 \mathrm{ml}$ of buffer $\mathrm{B}$ : 50 mM HEPES, $\mathrm{pH} 7.2,6.7 \%$ of supernatant containing myc-CD14 protein, $1.5 \mu \mathrm{g} \mathrm{ml}^{-1} \mathrm{SA}-\mathrm{XL} 665,5 \%$ fetal calf serum. In a 384 -well black plate, $10 \mu \mathrm{l}$ of buffer A, $9 \mu \mathrm{l}$ of buffer B and $1 \mu \mathrm{l}$ of sample solution were mixed and incubated for $16 \mathrm{~h}$ at $4{ }^{\circ} \mathrm{C}$. TR-FRET was measured with Discovery (Packard, Waltham, MA, USA) at an excitation of $337 \mathrm{~nm}$ and emission of $665 \mathrm{~nm}$. The inhibition rate $(\%)$ was calculated using the following formula.

Inhibition $(\%)=[1-\{$ (FRET ratio with compound $)-($ FRET ratio without LPS $)\} /\{$ (FRET ratio without compound $)-($ FRET ratio without LPS $)\}] \times 100$

\section{LPS stimulation and measurement of TNF- $\alpha$ production in the cell supernatants}

U937 cells were cultured in RPMI 1640 medium (Invitrogen) containing 10\% FBS at $37^{\circ} \mathrm{C}$ in a $5 \% \mathrm{CO}_{2}$ incubator. The cells were incubated in a 96-well plate at the density of $2 \times 10^{5}$ cells ml $^{-1}$ with $30 \mathrm{ng} \mathrm{ml}^{-1}$ of phorbol 12-myristate 13-acetate (PMA) for 3 days to differentiate them into macrophage-like cells. Then, the supernatants were removed and new media containing $5 \mathrm{ng} \mathrm{ml}^{-1}$ of LPS were added with or without test samples. After $4.5 \mathrm{~h}$, the supernatants were collected and the concentrations of TNF- $\alpha$ were measured using an HTRF kit (Cisbio) and Discovery (Packard).

\section{Detection of cytotoxicity}

HEK293 cells were seeded into 96-well plates in DMEM (Invitrogen) containing $10 \% \mathrm{FBS}$ and cultured overnight at $37^{\circ} \mathrm{C}$ in a $5 \% \mathrm{CO}_{2}$ in an incubator. Various concentrations of test samples were added and incubated for $20 \mathrm{~h}$. Then cell viability was monitored using Cell-Titer-Glo (Promega, Fitchburg, WI, USA).

\section{Determination of antimicrobial activity}

The minimum inhibitory concentrations (MICs) were determined by the medium microdilution method following the guidelines of the Clinical Laboratory Standards Institute. ${ }^{24}$

\section{ACKNOWLEDGEMENTS}

We would like to thank Ms Naoko Matsui and Dr Takao Ohyama for their support in the assay establishment. We acknowledge Dr Teiji Wada and Dr Fujio Isono for discussions and science review.

1 Hailman, E. et al. Lipopolysaccharide (LPS)-binding protein accelerates the binding of LPS to CD14. J. Exp. Med. 179, 269-277 (1994).

2 Bazil, V. et al. Biochemical characterization of a soluble form of the 53-kDa monocyte surface antigen. Eur. J. Immunol. 16, 1583-1589 (1986).

3 Haziot, A. et al. The monocyte differentiation antigen, CD14, is anchored to the cell membrane by a phosphatidylinositol linkage. J. Immunol. 141, 547-552 (1988).

4 Hoshino, K. et al. Cutting edge: Toll-like receptor 4 (TLR4)-deficient mice are hyporesponsive to lipopolysaccharide: evidence for TLR4 as the Lps gene product. J. Immunol. 162, 3749-3752 (1999).

5 Shimazu, R. et al. MD-2, a molecule that confers lipopolysaccharide responsiveness on Toll-like receptor 4. J. Exp. Med. 189, 1777-1782 (1999).

6 Da Silva Correia, J., Soldau, K., Christen, U., Tobias, P. S. \& Ulevitch, R. Lipopolysaccharide is in close proximity to each of the proteins in its membrane receptor complex: transfer from CD14 to TLR4 and MD-2. J. Biol. Chem. 276 , 21129-21135 (2001).

7 Beutler, B. \& Rietschel, E. T. Innate immune sensing and its roots: the story of endotoxin. Nat. Rev. Immunol. 3, 169-176 (2003).

8 Wong, Y. N. et al. Continuous pharmacodynamic activity of eritoran tetrasodium, a TLR4 antagonist, during intermittent intravenous infusion into normal volunteers. Innate. Immun. 14, 383-394 (2008).

9 Stansly, P. G., Shepherd, R. G. \& White, H. J. Polymyxin: a new chemotherapeutic agent. Bull. Johns Hopkins Hosp. 81, 43-54 (1947).

10 Rifkind, D. Prevention by polymyxin B of endotoxin lethality in mice. J. Bacteriol. 93 , 1463-1464 (1967).

11 Jacobs, D. M. \& Morrison, D. C. Inhibition of the mitogenic response to lipopolysaccharide (LPS) in mouse spleen cells by polymyxin B. J. Immunol. 118, 21-27 (1977).

12 From, A. H., Fong, J. S. \& Good, R. A. Polymyxin B sulfate modification of bacterial endotoxin: effects on the development of endotoxin shock in dogs. Infect. Immun. 23, 660-664 (1979). 
13 Vinnicombe, J. \& Stamey, T. A. The relative nephrotoxicities of polymyxin B sulfate sodium sulfomethyl-polymyxin B, sodium sulfomethyl-colistin (colymycin), and neomycin sulfate. Invest. Urol. 6, 505-519 (1969).

14 Mathis, G. Rare earth cryptates and homogeneous fluoroimmunoassays with human sera. Clin. Chem. 39, 1953-1959 (1993).

15 Hirota-Takahata, Y. et al. Pedopeptins, novel inhibitors of LPS: Taxonomy of producing organism, fermentation, isolation, physicochemical properties and structural elucidation. J. Antibiot. (doi:10.1038/ja.2013.122).

16 Huber, M. et al. R-form LPS, the master key to the activation of TLR4/MD-2-positive cells. Eur. J. Immunol. 36, 710-711 (2006).

17 Gangloff, S. C., Hijiya, N., Haziot, A. \& Goyert, S. M. Lipopolysaccharide structure influences the macrophage response via CD14-independent and CD14-dependent pathways. Clin. Infect. Dis. 28, 491-496 (1999).

18 Bryant, C. E. et al. The cellular Toll-like receptor 4 antagonist E5531 can act as an agonist in horse whole blood. Vet. Immunol. Immunopathol. 116, 182-189 (2007)
19 Zavascki, A. P. et al. Pharmacokinetics of intravenous polymyxin B in critically ill patients. Clin. Infect. Dis. 15, 1298-1304 (2008)

20 Mingeot-Leclercq, M. P., Tulkens, P. M., Denamur, S., Vaara, T. \& Vaara, M. Novel polymyxin derivatives are less cytotoxic than polymyxin $B$ to renal proximal tubular cells. Peptides 35, 248-252 (2012).

21 Vaara, M., Sader, S. S., Rhomberg, P. R., Jones, R. N. \& Vaara, T. Antimicrobial activity of the novel polymyxin derivative NAB739 tested against Gram-negative pathogens. J. Antimicrob. Chemother. 68, 636-639 (2013).

22 Tamaki, M. et al. Polycationic gramicidin S analogues with both high antibiotic activity and very low hemolytic activity. Chem. Pharm. Bull. 60, 1134-1138 (2012).

23 Hoess, A., Watson, S., Siber, G. R. \& Liddington, R. Crystal structure of an endotoxinneutralizing protein from the horseshoe crab, Limulus anti-LPS factor, at $1.5 \AA$ resolution. EMBO J. 12, 3352-3356 (1993).

24 National Committee for Clinical Laboratory Standards Methods for dilution antimicrobial susceptibility test for bacteria that grow aerobically, 5th ed., approved standard M7-A5 (NCCLS, Wayne, PA, USA, 2000). 